\title{
Anestesia regional en la paciente embarazada antiagregada o anticoagulada
}

\author{
Regional anesthesia in the antiplatelet or anticoagulated pregnant patient \\ Anestesia regional em paciente grávida antiplaquetária ou anticoagulada
}

Gimena dos Santos

ORCID: 0000-0002-9337-1833

Hematólogo. Asistente Cátedra de

Hematología. Hospital de Clínicas.

UdelaR.

Internista

Martín Pérez De Palleja

ORCID: 0000-0002-2935-4545

Anestesista. Profesor Adjunto Clínica

Anestesiología. Hospital de Clínicas.

UdelaR.

Viviana Castro

ORCID: 0000-0002-4348-0636

Médico. Cátedra de Hematología.

Hospital de Clínicas. UdelaR.

Paola Turcatti

ORCID: 0000-0002-4082-8246

Hematólogo

Médico especialista en Laboratorio

Clínico. Profesor Adjunto

Departamento de Laboratorio Clínico.

Hospital de Clínicas. UdelaR.

Resumen: La anestesia regional es la técnica de elección debida a la menor morbimortalidad. Una de las complicaciones más temidas es el hematoma espinal, teniendo las pacientes que reciben antiagregantes y/o anticoagulantes mayor riesgo de desarrollarlo. El cuadro clínico es variable, y no siempre están todos los elementos presentes (dolor lumbar, déficit motor y/o sensitivo y/o vesical). El diagnóstico se realiza por resonancia nuclear magnética y el tratamiento es la descompresión por laminectomía. Estando el pronóstico neurológico vinculado al tiempo en que se realiza ésta. Se realiza una revisión sobre los distintos fármacos antiagregantes y anticoagulantes utilizados en el embarazo - puerperio y las recomendaciones necesarias para las pacientes que recibirán una anestesia regional.

Palabras clave: anestesia regional, hematoma espinal, terapia antiagregante, terapia anticoagulante.

Abstract: Regional anesthesia is the technique of choice due to lower morbidity and mortality. One of the most feared complications is spinal hematoma, with patients receiving antiplatelet and / or anticoagulants having a greater risk of developing it. The clinical picture is variable, and not all the elements are always present (lumbar pain, motor and / or sensory and / or bladder deficits). Diagnosis is made by magnetic resonance imaging and treatment is laminectomy decompression. Being the neurological prognosis linked to the time in which it is performed. A review is made of the different antiplatelet and anticoagulant drugs used in pregnancy - puerperium and the necessary recommendations for patients who will receive regional anesthesia.

Key words: regional anesthesia, spinal hematoma, antiplatelet therapy, anticoagulant therapy.

Resumo: A anestesia regional é a técnica de escolha devido à menor morbimortalidade. Uma das complicações mais temidas é o hematoma espinhal, com pacientes em uso de antiagregantes plaquetários e / ou anticoagulantes com maior risco de desenvolvê-lo. O quadro clínico é variável e nem sempre os elementos estão presentes (dor lombar, déficits motores e / ou sensoriais e / ou vesicais). O diagnóstico é feito por ressonância magnética e o tratamento é a descompressão por laminectomia. Sendo o prognóstico neurológico ligado ao tempo em que é realizado. É feita uma revisão das diferentes drogas antiplaquetárias e anticoagulantes utilizadas na gravidez puerpério e as recomendações necessárias para as pacientes que receberão anestesia regional.

Palavras-chave: anestesia regional, hematoma espinhal, terapia antiplaquetária, terapia anticoagulante.

Recibido: 06/02/2021 - Aceptado: 31/05/2021 
Cada día son más las mujeres que requieren una intervención anestesiológica al final del embarazo. Esto se debe por un lado, al mayor acceso y disponibilidad de la analgesia del parto regional en sus diferentes modalidades (epidural o combinada espinal-epidural); por otro lado, al mayor número de nacimientos por cesárea, que constituye el procedimiento más frecuente en los block quirúrgicos y para el cual la anestesia regional es la técnica de elección debido a la menor morbimortalidad con respecto a la general ${ }^{1}$.

Dentro de las complicaciones más temidas de esta técnica se encuentra el hematoma espinal (HE), que puede localizarse en el espacio peridural, subdural o subaracnoideo y puede determinar compresión de estructuras nerviosas con alto riesgo de secuelas permanentes.

Las pacientes que reciben antiagregantes $y / 0$ anticoagulantes y son candidatas a anestesia regional tienen mayor riesgo de desarrollar HE, por lo que la apliación de estas técnicas deben seguir un criterio riguroso de riesgo/beneficio.

EI HE tuvo una incidencia históricamente baja hasta la década del noventa en que tras la incorporación de las heparinas de bajo peso molecular (HBPM) se produce un alarmante aumento de su incidencia.

De $1 \mathrm{HE}$ cada 150.000 - 200.000 técnicas regionales se incrementó a $1 \mathrm{HE}$ cada 3.000-5.000 en la población sometida a cirugía ortopédica. En el Third National Audit Project del Colegio de Anestesiólogos del Reino Unido se reportó una incidencia en anestesia neuroaxial de 0.85 por 100.000 procedimientos $^{2}$.

Los factores de riesgo para el desarrollo de un HE son los siguientes:

4) Coagulopatías (ya sea por patologías o por efecto farmacológico).

5) Técnica regional utilizada: la técnica más frecuentemente involucrada fue la peridural continua con catéter. El aumento de la incidencia secundaria a la técnica peridural, no sólo se explica por el uso de agujas de mayor diámetro, sino también por el uso de catéteres peridurales que quedan ubicados en un lugar que posee abundantes plexos venosos. Los catéteres peridurales no sólo pueden traumatizar vasos directamente, sino que también originan desprendimiento de coágulos, secundarios a pequeños desplazamientos, lo que puede aumentar el riesgo de hematoma en pacientes con alteración de la hemostasia. Es importante destacar que casi la mitad de los hematomas aosciados a anestesia peridural continua, han ocurrido en relación al retiro del catéter, por lo que se establece que este evento no es del todo inocuo, y es de exclusiva responsabilidad del anestesiólogo.

6) Punción traumática (hemorrágica): punción dificultosa.

7) Patologías o anormalidades anatómicas: la patología en sí, no constituye un mayor riesgo, pero sí el hecho de realizar una punción más traumática. En pacientes que están bajo acción de anti-inflamatorios y que a la vez poseen un canal medular más estrecho, es posible producir una compresión medular más precozmente.

8) Edad avanzada

9) Sexo femenino

El cuadro clínico de un HE es variable, ya que el orden de aparición de los síntomas y/o signos no siempre es igual, y no siempre están todos presentes: dolor lumbar tipo radicular que generalmente es muy intenso, y no cede con medidas analgésicas habituales, déficit motor, déficit sensitivo, disfunción vesical.

El diagnóstico se realiza por resonancia nuclear magnética. El tratamiento es la descompresión por laminectomía. Los resultados neurológicos dependen de la rapidez con que se produzca la compresión, del tamaño y extensión del hematoma, del compromiso neurológico existente y fundamentalmente del tiempo transcurrido. La probabilidad de reversión completa es mayor si la descompresión ocurre en las primeras 8 horas de instalados los síntomas ${ }^{3}$.

\section{Terapia antiplaquetaria}

\section{a) Ácido Acetil-Salicílico (AAS) y Antiinflamatorios no esteroideos (AINEs)}

Múltiples estudios han demostrado la relativa seguridad del bloqueo neuroaxial en combinación con el tratamiento antiplaquetario. 
EI CLASP (Collaborative Low-Dose Aspirin Study in Pregnancy) incluyó 1422 pacientes obstétricas de alto riesgo a las que se les administró $60 \mathrm{~g}$ de AAS al día y se sometieron a anestesia epidural sin secuelas neurológicas ${ }^{4}$.

Los estudios de Horlocker et al, de aproximadamente 1000 pacientes en cada estudio, no mostraron $\mathrm{HE}$, aunque se observó sangre durnate la colocación del catéter o la aguja, en el $22 \%$ de las pacientes ${ }^{5,6}$.

Un estudio posterior en pacientes que se encontraban en tratamiento con AINEs y se sometieron a bloqueo neuroaxial no desarrollaron hematoma intraespinal ${ }^{7}$.

Se concluye entonces que el bloqueo neuroaxial puede realizarse de manera segura en los pacientes que toman AAS o AINEs y en pacientes que toman inhibidores de la ciclooxigenasa $2^{8}$.

Las recomendaciones del Departamento de Anestesiología de la UdelaR basadas en las guías Sociedad Americana de Anestesia Regional consideran que la asociación de anestesia/ analgesia neuroaxial e inhibidores de la enzima ciclooxigenasa 2 no constituye mayor riesgo para $\mathrm{HE}$, ya sea anestesia raquídea o peridural, por lo que el uso de AAS no contraindica las técnicas regionales anestesiológicas ${ }^{8}$.

Las guías provenientes de sociedades europeas son un poco más rígidas y sugieren por lo menos un intervalo libre de AAS de tres días antes de realizar anestesia neuroaxial o retiro de catéteres epidurales post cirugía o parto.

Las pacientes que están bajo efecto de AAS u otros AINEs y además reciben otro fármaco que altere la coagulación en el período perioperatorio, no deberían recibir anestesia/analgesia neuroaxial ya que se potencia el efecto antiagregante y existe mayor riesgo de HE (grado 2C).

\section{b) Otros Antiplaquetarios (categoría B en embarazo)}

En caso excepcional de uso de Clopidogrel o Ticlopidina, se sugiere realizar anestesia/ analgesia neuroaxial una vez recuperada la función plaquetaria, lo que se logra suspendiendo ticlopidina por 10- 14 días y clopidogrel por 7 días. Si una paciente está bajo acción de clopidogrel y se desea realizar anestesia/analgesia neuroaxial entre los 5 y 7 días de suspendido el fármaco se debería documentar la normalización de la función plaquetaria (grado 1C).

\section{Terapia anticoagulante}

\section{a) HBPM en el período preoperatorio}

Si una paciente está bajo acción de HBPM en el período preoperatorio debe asumirse una alteración de la coagulación y no debería practicarse anestesia/analgesia neuroaxial hasta pasadas 12 horas de la última dosis profiláctica y 24 horas si recibe dosis terapéuticas (enoxaparina $1 \mathrm{mg} / \mathrm{Kg}$ cada 12 horas, enoxaparina 1,5 mg/Kg al día) (grado 1C).

No debería realizarse anestesia/analgesia neuroaxial si se ha administrado HBPM 2 horas antes, ya que coincide con el pico de su concentración plasmática ${ }^{8}$ (grado $1 \mathrm{~A}$ ).

Con respecto a la técnica elegida, las guías no hacen mención a técnicas continuas en pacientes con administración de HBPM en el período preoperatorio, sugiriendo realizar anestesia en dosis únicas (anestesia raquídea).

\section{b) HBPM en el período postoperatorio}

Pueden recibir anestesia/analgesia neuroaxial en forma más segura, ya sea técnicas de dosis únicas como técnicas continuas. Las guías se rigen según el esquema de terapia involucrado 9,10:

- Esquema de dos dosis al día: En los pacientes que reciben HBPM a dosis de anticoagulación, la primer dosis en el post operatorio no debe de administrarse antes de 4 horas de remover el catéter y como mínimo no antes de 24 horas luego de haberse colocado el mismo (American Society of Regional Anesthesia; 2018).

El grupo de trabajo de las presentes guías entiende que en situaciones de alto riesgo (pacientes con válvulas mecánicas o con eventos trombóticos en el último mes) en las que extender el tiempo de inicio de anticoagulación puede someter a la paciente a una recurrencia 
o a un nuevo evento trombótico, deberá valorarse en conjunto con la paciente, la familia y el equipo tratante iniciar la primer dosis de anticoagulación previo a los plazos referidos. En caso que no fuera posible acortar los plazos, deberá evaluarse otra vía de anestesia.

- Esquema de una dosis diaria: cuando la HBPM se administra una sola vez al día, la primera dosis puede darse 6 a 8 horas después de realizada la anestesia/analgesia neuroaxial. Los catéteres pueden mantenerse en forma más segura, teniendo presente que deben retirarse 10 a 12 horas después de la última dosis de heparina. Luego de retirado el catéter peridural se esperará 4 horas para reiniciar la heparina de bajo peso molecular (grado 1C).

Las pacientes bajo acción de otro fármaco con efecto anticoagulante como antiplaquetarios o anticoagulantes orales (excepcional en la situación del embarazo) y que además reciben HBPM poseen mayor riesgo de HE, por lo que se recomienda NO realizar anestesia/analgesia neuroaxial en este grupo de pacientes, independientemente del esquema o dosis de HBPM administrada (grado $1 \mathrm{~A}$ ).

Independientemente del esquema involucrado las pacientes que reciben punciones traumáticas tienen mayor riesgo de $\mathrm{HE}$, por lo que el inicio de la terapia debería ser postergada por 24 hrs (grado 2C).

\section{c) Heparina no fraccionada (HNF) en el período preoperatorio}

Si la paciente está recibiendo HNF intravenosa la misma debe ser suspendida por lo menos 4hs previas y tener un control normal de TTPa antes de la realización de las técnicas regionales 8. Recomendación IA.

\section{d) HNF en el período postoperatorio}

Se puede reincorporar luego de $1 \mathrm{~h}$ de realizada la técnica regional o la colocación del catéter peridural siempre que la punción no sea hemática. Si la punción es hemática se recomienda esperar $2 \mathrm{hs}^{8}$. Recomendación IA.

En la tabla 1 se presentan las recomendaciones según el fármaco.

Tabla 1: Recomendaciones para anestesia-analgesia neuroaxial según el

\begin{tabular}{|l|l|l|}
\multicolumn{1}{|c|}{ Fármaco } & $\begin{array}{r}\text { Recomendación para anestesia- analgesia } \\
\text { neuroaxial }\end{array}$ & \multicolumn{1}{c|}{ Observaciones } \\
\hline AAS & Sin contraindicaciones & $\begin{array}{l}\text { Guías europeas sugieren suspender } 3 \text { días } \\
\text { previos }\end{array}$ \\
\hline HBPM profiláctica & Suspender 12 hs antes & Retomar 12 hs después \\
\hline HBPM terapéutica & Suspender 24 hs antes & Retomar 24 hs después \\
\hline
\end{tabular}

\section{Bibliografía}

1- Alvarez C, Illescas L, Pérez Palleja M. Perioperatorio Operación Cesarea. Perioper. y Analg. Reg. Obs. $2017 ; 134$.

2- Cook TM, Counsell D, Wildsmith JAW. Major complications of central neuraxial block: Report on the Third National Audit Project of the Royal College of Anaesthetists. Br. J. Anaesth. 2009;102(2):179-190.

3- Perez De Palleja M, Stevenazzi M. Enfermedad tromboembolica venosa al final de embarazo y puerperio. Perioper. y Analg. Reg. Obs. 2017;421.

4- CLASP: a randomised trial of low-dose aspirin for the prevention and treatment of pre-eclampsia among 9364 pregnant women. CLASP (Collaborative Low-dose Aspirin Study in Pregnancy) Collaborative Group. Lancet. 1994 Mar 12;343(8898):619-29.

5- Horlocker TT, Wedel DJ, Offord KP. Does preoperative antiplatelet therapy increase the risk of hemorrhagic complications associated with regional anesthesia? Anesth. Analg. 1990;70(6):631-634.

6- Horlocker TT, Wedel DJ, Schroeder DR, Rose SH, Elliott BA, McGregor DG, et al. Preoperative antiplatelet therapy does not increase the risk of spinal hematoma associated with regional anesthesia. Anesth. Analg. 1995;80(2):303-309.

7- Horlocker TT, Bajwa ZH, Ashraf Z, Khan S, Wilson JL, Sami N, et al. Risk assessment of hemorrhagic complications associated with nonsteroidal antiinflammatory medications in ambulatory pain clinic patients undergoing epidural steroid injection. Anesth. Analg. 2002;95(6):1691-1697. 
8- Horlocker TT, Vandermeuelen E, Kopp SL, Gogarten W, Leffert LR, Benzon HT. Regional Anesthesia in the Patient Receiving Antithrombotic or Thrombolytic Therapy: American Society of Regional Anesthesia and Pain Medicine Evidence-Based Guidelines (Fourth Edition). Reg. Anesth. Pain Med. 2018;43(3):263-309.

9- Horlocker TT, Wedel DJ, Rowlingson JC, Enneking FK, Kopp SL, Benzon HT, et al. Regional anesthesia in the patient receiving antithrombotic or thrombolytic therapy: American Society of Regional Anesthesia and Pain Medicine Evidence-Based Guidelines (Third Edition). Reg. Anesth. Pain Med. 35(1):64-101.

10- Gogarten W, Vandermeulen E, Van Aken H, Kozek S, Llau JV, Samama CM; European Scoeity of Anaesthesiology. Regional anaesthesia and antithrombotic agents: recommendations of the European Society of Anaesthesiology. Eur J Anaesthesiol 2010; 27:999.

\section{Aportes de cada autor al trabajo}

Gimena dos Santos: Concepción y diseño del trabajo, recolección de datos, redacción y revisión crítica del manuscrito.

Martín Pérez De Palleja: Concepción y diseño del trabajo, recolección de datos, redacción y revisión crítica del manuscrito.

Viviana Castro: Concepción y diseño del trabajo, recolección de datos, redacción y revisión crítica del manuscrito.

Paola Turcatti: Concepción y diseño del trabajo, recolección de datos, redacción y revisión crítica del manuscrito. 